\title{
Chemical enrichment in Ultra-Faint Dwarf galaxies
}

\author{
Donatella Romano
}

INAF, Osservatorio Astronomico di Bologna, Via Ranzani 1, I-40127, Bologna, Italy

email: donatella.romano@oabo.inaf.it

\begin{abstract}
Our view of the Milky Way's satellite population has radically changed after the discovery, ten years ago, of the first Ultra-Faint Dwarf galaxies (UFDs). These extremely faint, dark-matter dominated, scarcely evolved stellar systems are found in ever-increasing number in our cosmic neighbourhood and constitute a gold-mine for studies of early star formation conditions and early chemical enrichment pathways. Here we show what can be learned from the measurements of chemical abundances in UFD stars read through the lens of chemical evolution studies, point out the limitations of the classic approach, and discuss the way to go to improve the models.
\end{abstract}

Keywords. Galaxies: dwarf, galaxies: evolution, galaxies: individual: Boötes I, Segue 1, stars: abundances

\section{Introduction}

The past two decades have seen the advent of the era of large-scale digital sky surveys, which has had a dramatic impact on studies of the local universe (Ivezic et al. 2012). In particular, since 2005 the Sloan Digital Sky Survey (SDSS; York et al. 2000) has more than doubled the number of known Milky Way satellites, alleviating - if not providing a way out of - the 'missing satellite' problem (Klypin et al. 1999; Moore et al. 1999; see Tollerud et al. 2008). Nowadays deeper surveys are being conducted (the SkyMapper Southern Sky Survey, the Dark Energy Survey, Pan-STARRS...), that will greatly improve the areal coverage and eventually deliver a complete census of UFDs out to the Milky Way virial radius. This will set more stringent constraints to large-scale structure formation theories. In the meanwhile, the brightest giant star members of the newlydiscovered satellites are promptly targeted for spectroscopy in order to shed light on a number of questions: did these small, sparse stellar systems suffer 'one-shot' or extended star formation? what is the level and significance of chemical inhomogeneity inside them? was stellar feedback effective in removing all the gas left over from the star formation process, or did the interaction with the environment play a major role? are the chemical properties of UFDs consistent with the idea that they are the surviving relics of the accretion processes that shaped the diffuse Milky Way halo in hierarchical theories of galaxy formation?

As for the last question, it has been noted that the cumulative metallicity distribution function (MDF) for the metal-poor tails of a number of UFDs well compares to that of the Milky Way halo (Kirby et al. 2008). Moreover, a significant number of UFD stars with $[\mathrm{Fe} / \mathrm{H}]<-2.5$ have $[\alpha / \mathrm{Fe}]$ ratios consistent with those of halo stars of similar metallicity, within the uncertainties (Vargas et al. 2013). This would indicate that the UFDs are good candidates for the basic building blocks from which the halo was assembled. However, the abundances of neutron-capture elements in UFDs do not follow the typical halo 
Table 1. Overview of currently available high-resolution spectroscopic data for UFD giants.

\begin{tabular}{lccc}
\hline Object name & Instrument@Telescope & $\begin{array}{c}\text { \# of analysed } \\
\text { member stars }\end{array}$ & Reference \\
\hline Boötes I & HIRES@Keck & 7 & Feltzing et al. $(2009)$ \\
& UVES@VLT & 8 & Gilmore et al. $(2013)$ \\
Ursa Major II & HDS@Subaru & 6 & Ishigaki et al. $(2014)$ \\
Coma Berenices & HIRES@Keck & 3 & Frebel et al. $(2010)$ \\
Hercules & HIRES@Keck & 3 & Frebel et al. $(2010)$ \\
Leo IV & MIKE@Magellan & 2 & Koch et al. $(2008)$ \\
Segue 1 & MIKE@Magellan & 1 & Simon et al. $(2010)$ \\
Boötes II & HIRES@Keck & 6 & Frebel et al. $(2014)$ \\
Segue 2 & MIKE@Magellan & 1 & Koch \& Rich $(2014)$ \\
& & 1 & Roederer \& Kirby $(2014)$ \\
\hline
\end{tabular}

Note: last update July 31, 2015.

abundance pattern in the metallicity range $-3.0 \leqslant[\mathrm{Fe} / \mathrm{H}] \leqslant-2.5$ (Koch et al. 2008; Frebel et al. 2010), making the identification with the halo building blocks less straightforward (see also complementary arguments by Giuliana Fiorentino, these proceedings).

It is clear that, in order to unravel the UFDs' chemical enrichment history and, thus, (at least a piece of) the halo assembly history, one has to fully exploit the information encoded in the abundances of different chemical elements, produced by different stellar factories on different timescales. To do this, high-resolution spectroscopic observations in UFDs are badly needed. Unfortunately, high-resolution spectroscopy in these objects is very challenging and only a few stars per galaxy have been observed so far (see Table 1). In the following, I will mostly focus on Boötes I. Located at $66 \pm 2 \mathrm{kpc}$ distance from the Sun and with an absolute visual magnitude of $M_{V}=-6.3 \pm 0.2 \mathrm{mag}$ (McConnachie 2012), it is one of the brightest UFDs orbiting the Milky Way and it has the largest number of high-resolution abundance determinations, as well as a well-defined MDF (Lai et al. 2011).

\section{Chemical evolution models}

In Romano et al. (2015) we present one-zone chemical evolution models for Boötes I. The usual set of integro-differential equations (Talbot \& Arnett 1971; Tinsley 1980; Matteucci 2001) is solved numerically and the evolution of the average abundances of several chemical elements in the interstellar medium (ISM) is followed by taking into account in detail the stellar lifetimes (no instantaneous recycling approximation). The adopted stellar yields are tested against the Milky Way data and shown to reproduce reasonably well the abundance patterns of several elements (Romano et al. 2010).

We run classic models, resting on a number of assumptions: (i) smooth infall of gas of primordial chemical composition provides the raw material for star formation; (ii) the stellar ejecta mix instantaneously with the ISM; (iii) galactic outflows develop when the thermal energy of the gas heated by supernova (SN) explosions exceeds its binding energy; (iv) the stellar initial mass function (IMF; Kroupa 2001) is constant in space and time. We also run cosmologically-motivated models, where we adopt mass assembly histories of candidate Boötes I-like galaxies selected from the mock satellite catalogue of Starkenburg et al. (2013). These were obtained by coupling the Munich semi-analytical model of galaxy formation ( $\mathrm{Li}$ et al. 2010, and references therein) to the Aquarius dark matter simulations (Springel et al. 2008). According to Li et al. (2010) and Starkenburg et al. (2013), only part of the stellar ejecta is directly mixed to the cold ISM, while 95 per cent of the newly-produced metals are recycled through the hot galaxy's halo. Furthermore, the matter expelled through the outflow is stored in an 'ejected gas' component and may 

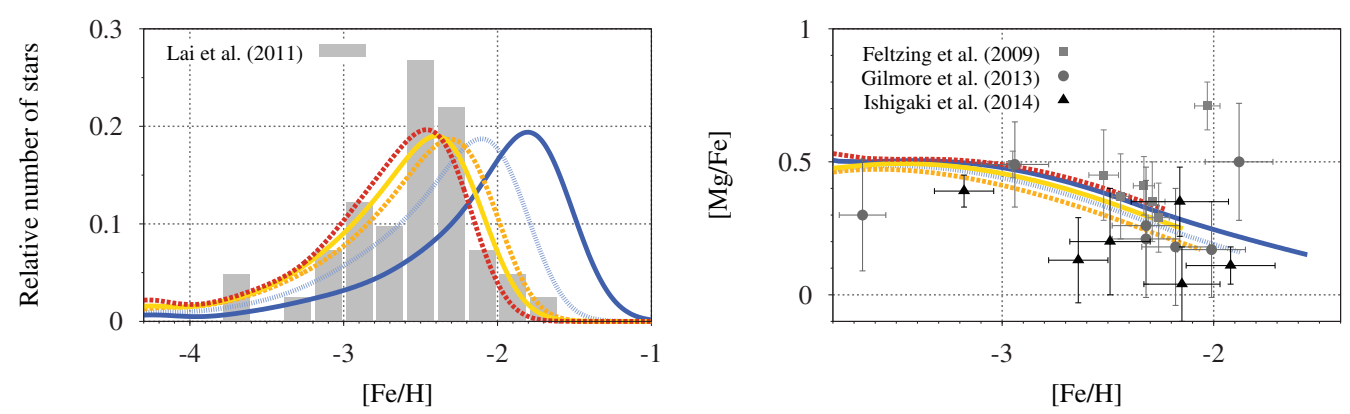

Figure 1. Left panel: theoretical (curves) and observed (histogram) MDFs of Boötes I. Right panel: theoretical (curves) and observed (symbols) $[\mathrm{Mg} / \mathrm{Fe}]$ versus $[\mathrm{Fe} / \mathrm{H}]$ relations for Boötes I. The high $[\mathrm{Mg} / \mathrm{Fe}]=0.71$ measured by Feltzing et al. (2009) for the star Boo-127 has been revised downwards by both Gilmore et al. (2013) and Ishigaki et al. (2014).

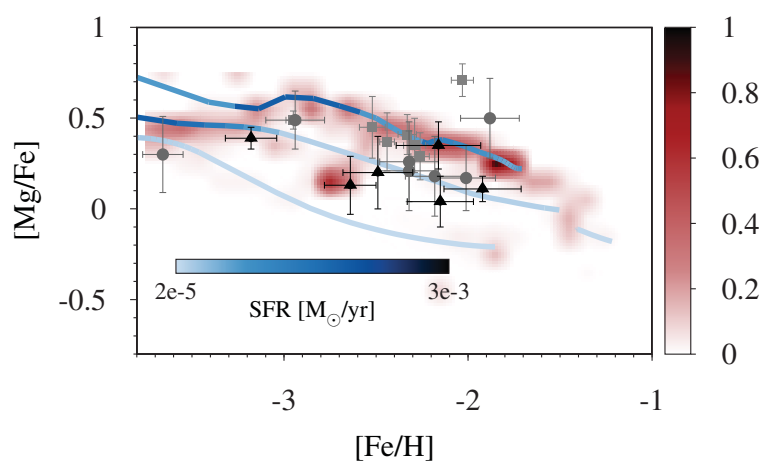

Figure 2. $[\mathrm{Mg} / \mathrm{Fe}]$ versus $[\mathrm{Fe} / \mathrm{H}]$ relations for the models run in a fully cosmological context. The density map shows the distribution of synthetic long-lived stars for 11 Boötes I candidates in the mock Milky Way satellite catalogue of Starkenburg et al. (2013) normalised to its maximum value. The curves show the predictions of three representative models and are colour-coded according to the strength of the star formation rate (see legend on the bottom of the plot).

eventually be re-accreted by the galaxy. We include all these gas flows in our models. Last but not least, the processes of stripping are included in the cosmologically-motivated models, while they are neglected in the classical models.

\subsection{A small fish in a big pond?}

In Fig. 1 we show the MDF (left panel) and $[\mathrm{Mg} / \mathrm{Fe}]$ versus $[\mathrm{Fe} / \mathrm{H}]$ relation of Boötes I stars (right panel) predicted by our classic chemical evolution model (lines) with different assumptions about the duration and efficiency of star formation, as well as about the dark and baryonic matter content of the galaxy (see Romano et al. 2015 for details on the model parameters). The model predictions are compared to the observations (legend on the top-left corner of each plot). Similarly, Fig. 2 shows the predictions of the fully cosmological models. In general, a good agreement between model predictions and data is found by assuming a very low efficiency of conversion of gas into stars, which confirms previous results for UFDs by Salvadori \& Ferrara (2009) and Vincenzo et al. (2014). Successful models, in fact, require that $\sim 10^{7} \mathrm{M}_{\odot}$ of gas are present at the epoch of star formation to dilute the metals produced by SN explosions; out of these, only $\sim 6 \times 10^{4} \mathrm{M}_{\odot}$ end up as long-lived stars that still inhabit the galaxy (value of present-day stellar mass from McConnachie 2012). We do not find any clearcut evidence that galactic winds 


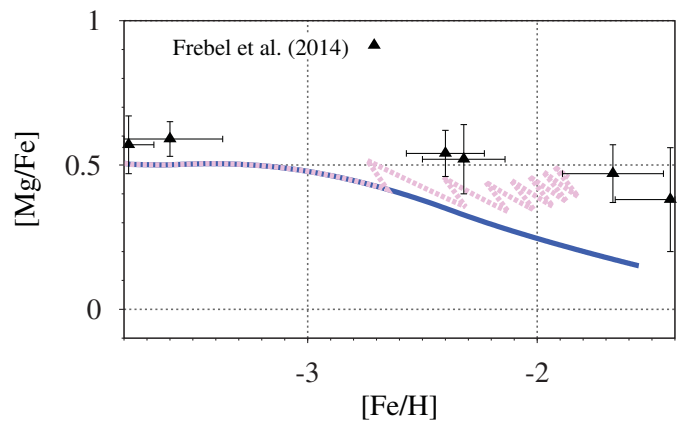

Figure 3. $[\mathrm{Mg} / \mathrm{Fe}]$ versus $[\mathrm{Fe} / \mathrm{H}]$ behaviour in Segue 1. The triangles are high-resolution spectroscopic observations of giant stars by Frebel et al. (2014). The lines are the predictions of two chemical evolution models. The duration of the star formation is set to 1 Gyr for both models, but in one case (dashed line) it is assumed that the ejecta from SNeIa is vented out of the galaxy much more efficiently than that from SNeII.

deprive the galaxy of all its residual gas; since no neutral gas is associated to Boötes I today, we conclude that stripping must have played a fundamental role.

The need for a substantially more massive progenitor can be easily understood. If each type II SN (SNII) produces $0.07 \mathrm{M}_{\odot}$ of Fe that fully mix within the considered volume, in the absence of metal-rich outflows $<2$ SNII explosions suffice to increase the metal content of a $10^{5} \mathrm{M}_{\odot}$ gas cloud from $[\mathrm{Fe} / \mathrm{H}] \simeq-4$ to $\simeq-3$, while a metallicity $[\mathrm{Fe} / \mathrm{H}] \simeq-2$ is reached after $\sim 20$ such events. Since up to $700 \mathrm{SNeII}$ are expected to have exploded in Boötes I (when considering a present-day mass in stars of $\sim 6 \times 10^{4} \mathrm{M}_{\odot}$ and a canonical IMF), we end up with the request that some $10^{6} \mathrm{M}_{\odot}$ of gas must have been present to dilute the freshly-produced metals and not to shift the peak of the MDF towards values higher than observed (Fig. 1, left panel). Since some tens of SNeIa are also expected to have polluted the ISM while Boötes I was still forming stars (see Romano et al. 2015, their figure 6), even more diluting gas is needed. It is worth stressing that our conclusion that Boötes I must have been much more massive in the past is based mainly on the assumption of a canonical IMF (Kroupa 2001): the steeper the IMF slope in the high-mass regime, the lower the number of core-collapse SNe and, hence, the lower the gas amount necessary to dilute the ejecta.

\subsection{Star formation histories and chemical enrichment}

Recently, Brown et al. (2014) combined photometric and spectroscopic data for five UFDs and found that these galaxies formed the bulk of their stars long ago, by $z \sim 6$ (12.8 Gyr ago). They did not experience any significant star formation afterwards, most likely because of the quenching effects of reionization. In principle, one might try to put more stringent constraints on the duration of the star formation in UFDs by using chemical evolution arguments. For instance, the occurrence of a knee in the $[\alpha / \mathrm{Fe}]$ versus $[\mathrm{Fe} / \mathrm{H}]$ relation can be interpreted as an indication for a significant contribution to the chemical evolution from SNeIa and the $[\mathrm{Fe} / \mathrm{H}]$ value where the knee occurs related to the system's formation timescale (Matteucci \& Brocato 1990, their figure 4). In practice, we analyse models for Boötes I where the bulk of the stellar population forms in $\sim 100 \mathrm{Myr}$ or in $\sim 1$ Gyr and find that all can fit the data reasonably well after a proper fine-tuning of the free parameters; the degeneracy is mainly due to the approximations introduced to treat the galactic outflows (see Romano et al. 2015, for a discussion).

Fig. 3 further illustrates this problem, by showing the predictions of two different models for the UFD Segue 1. This galaxy displays an almost constant $[\mathrm{Mg} / \mathrm{Fe}]$ ratio in the 


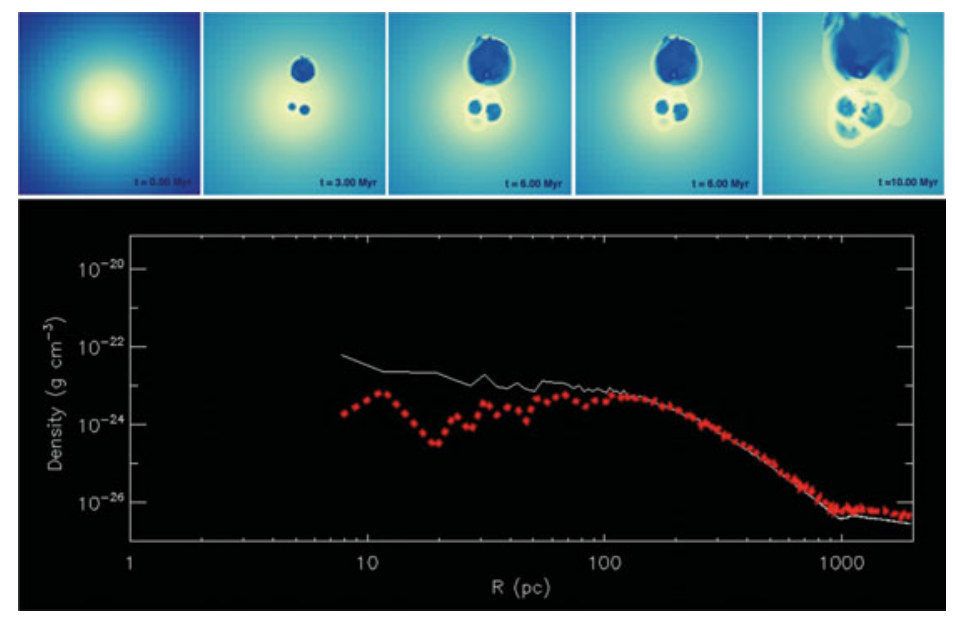

Figure 4. Top: gas density maps in the XY plane for the simulated Boötes I UFD at different times from 0 to $10 \mathrm{Myr}$, as labeled in the bottom right corner of each snapshot. The simulation uses a box size of $2 \mathrm{kpc}$. The maximal refinement level is set by $l_{\max }=11$, which corresponds to a sub-parsec $(0.9 \mathrm{pc})$ maximal spatial resolution. Bottom: gas density profiles at $t=0 \mathrm{Myr}$ (solid line) and $t=10 \mathrm{Myr}$ (dashed line). After $10 \mathrm{Myr}$ from the beginning of the simulation, the gas starts to be pushed towards the external regions, because of the energy release by multiple SNe exploding in associations.

metallicity range $-4<[\mathrm{Fe} / \mathrm{H}]<-1.5$ (Frebel et al. 2014) - a clear signature of chemical enrichment from core-collapse SNe alone. In the framework of classic chemical evolution models, this is interpreted as an indication for a short-lasting star formation, of the order of $30 \mathrm{Myr}$ or so. In fact, if the star formation lasts longer, a knee appears, due to Fe production from SNeIa (Fig. 3, solid line). However, if the products of SNIa nucleosynthesis are expelled from the galaxy before mixing with the ISM and more efficiently than SNII ejecta, the $[\mathrm{Mg} / \mathrm{Fe}]$ ratio in the galaxy may be kept flat on timescales as long as $\sim 1$ Gyr (Fig. 3, dashed line). Such an assumption may be justified on the basis of 2D hydrodynamical simulations by Recchi et al. (2001), who found that the ejecta of SNeIa, exploding in a medium already rarefied by previous SNII explosions, find an easier way out of the galaxy.

\section{Future work}

Chemical evolution models have succeeded in explaining many observed properties of galaxies. However, when moving to stellar systems with lower and lower dynamical masses, severe uncertainties affect the model predictions: the treatment of mechanical feedback from stars, the conditions set for the development of large-scale outflows, the assumed mass loading factors of the ISM cause a high degeneracy of the proposed solutions. In order to make a quantum leap forward in our understanding of the formation and evolution of systems with very shallow potential wells, detailed chemical evolution models must be coupled self-consistently to 3D hydrocodes. To this aim, we have recently started to study the evolution of a galaxy resembling Boötes I by means of a customised version of the adaptive mesh refinement code RAMSES (Teyssier 2002). In Fig. 4 we show some preliminary results of our simulation, to be presented in a forthcoming paper. 


\section{Acknowledgements}

I thank my collaborators M. Bellazzini, F. Calura, C. G. Few, A. D'Ercole, R. Leaman and E. Starkenburg. I acknowledge financial support from the IAU and from the Italian Ministero dell'Istruzione, dell'Università e della Ricerca (MIUR) through the grant 'The Chemical and Dynamical Evolution of the Milky Way and Local Group Galaxies', prot. 2010LY5N2T. The simulations discussed in this contribution are performed on the Galileo supercomputer at CINECA (Bologna, Italy), within projects supported by the Italian SuperComputing Resource Allocation (ISCRA).

\section{References}

Brown, T. M., et al. 2014, ApJ, 796, 91

Feltzing, S., Eriksson, K., Kleyna, J., \& Wilkinson, M. I. 2009, A\&3A (Letters), 508, L1

Frebel, A., Simon, J. D., Geha, M., \& Willman, B. 2010, ApJ, 708, 560

Frebel, A., Simon, J. D., \& Kirby, E. N. 2014 ApJ, 786, 74

Gilmore, G., et al. 2013, ApJ, 763, 61

Ishigaki, M. N., Aoki, W., Arimoto, N., \& Okamoto, S. 2014, Aध A, 562, A146

Ivezić, Ž., Beers, T. C., \& Jurić, M. 2012, ARAA, 50, 251

Kirby, E. N., et al. 2008, ApJ (Letters), 685, L43

Klypin, A., Kravtsov, A. V., Valenzuela, O., \& Prada, F. 1999, ApJ, 522, 82

Koch, A., et al. 2008, ApJ (Letters), 688, L13

Koch, A. \& Rich, R. M. 2014, ApJ, 794, 89

Kroupa, P. 2001, MNRAS, 322, 231

Lai, D. K., et al. 2011, ApJ, 738, 51

Li, Y.-S., De Lucia, G., \& Helmi, A. 2010, MNRAS, 401, 2036

Matteucci, M. 2001, The Chemical Evolution of the Galaxy (Dordrecht: Kluwer)

Matteucci, M. \& Brocato, E. 1990, ApJ, 365, 539

McConnachie, A. W. 2012, AJ, 144, 4

Moore, B., et al. 1999, ApJ (Letters), 524, L19

Recchi, S., Matteucci, F., \& D'Ercole, A. 2001, MNRAS, 322, 800

Roederer, I. U. \& Kirby, E. N. 2014, MNRAS, 440, 2665

Romano, D., Bellazzini, M., Starkenburg, E., \& Leaman, R. 2015, MNRAS, 446, 4220

Romano, D., Karakas, A. I., Tosi, M., \& Matteucci, F. 2010, A\&A, 522, A32

Salvadori, S. \& Ferrara, A. 2009, MNRAS (Letters), 395, L6

Simon, J. D., Frebel, A., McWilliam, A., Kirby, E. N., \& Thompson, I. B. 2010, ApJ, 716, 446

Springel, V., et al. 2008, MNRAS, 391, 1685

Starkenburg, E., et al. 2013, A\&\&A, 549, A88

Talbot, R. J., Jr. \& Arnett, W. D. 1971, ApJ, 170, 409

Teyssier, R. 2002, A\& A, 385, 337

Tinsley, B. M. 1980, Fundam. Cosm. Phys., 5, 287

Tollerud, E. J., Bullock, J. S., Strigari, L. E., \& Willman, B. 2008, ApJ, 688, 277

Vargas, L. C., Geha, M., Kirby, E. N., \& Simon, J. D. 2013, ApJ, 767, 134

Vincenzo, F., Matteucci, F., Vattakunnel, S., \& Lanfranchi, G. A. 2014, MNRAS, 441, 2815

York, D. G., Adelman, J., Anderson, J. E., et al. 2000, AJ, 120, 1579 